\title{
Preliminary study on qualitative and quantitative detection of norfloxacin based on terahertz spectroscopy
}

\author{
Long Yuan ${ }^{1,2}$, Li Bin ${ }^{1,2,3 *}$ \\ (1. Beijing Research Center for Information Technology in Agriculture, Beijing 100097, China; \\ 2. Key Laboratory of Agri-informatics, Ministry of Agriculture, Beijing 100097, China; \\ 3. Beijing Key Laboratory of Digital Plant, Beijing 100097, China)
}

\begin{abstract}
Norfloxacin is a kind of quinolone bactericide which is widely used in food industry, livestock feed and medicine area. At present, how to develop a rapid, convenient and accurate technique for qualitative and quantitative detection of norfloxacin is still a challenge. In this study, Terahertz Time-domain Spectroscopy technology (THz-TDS) was used to explore qualitative and quantitative analysis of norfloxacin. Forty-five norfloxacin samples with different levels were prepared. The pure norfloxacin powder was mixed with polyethylene (PE) powder together and made into tablets by tablet-making machine. The minimum detection concentration was $10 \%$ and the maximum detection concentration evaluated in the paper was $90 \%$. Then, terahertz (THz) spectra of each sample were measured on K15 THz-TDS equipment. The multiple linear regressions and the partial least squares regression algorithms were used to establish a model to make quantitative analysis. From the results, two typical fingerprint peaks of norfloxacin in terahertz band were observed at 0.944 $\mathrm{THz}$ and $1.206 \mathrm{THz}$, and the correlation coefficient and the root mean square error of the established model reached 0.9908 and 0.0481, respectively, which showed that the model performed well for the prediction. This preliminary study indicates that $\mathrm{THz}$ spectroscopy has great potential for future screening applications to detect the presence of norfloxacin residues in food, livestock feed and medicine industries.
\end{abstract}

Keywords: Terahertz spectroscopy, norfloxacin, qualitative and quantitative detection, model, correlation coefficient DOI: $10.25165 /$ j.jijabe.20171005.2962

Citation: Long Y, Li B. Preliminary study on qualitative and quantitative detection of norfloxacin based on terahertz spectroscopy. Int J Agric \& Biol Eng, 2017; 10(5): 262-268.

\section{Introduction}

Terahertz time-domain spectroscopy (THz-TDS) is the wavelength band of electromagnetic radiation waves observed in the millimeter range of $0.1-30 \mathrm{THz}^{[1]}$, which is very sensitive to macromolecular substances. When a terahertz light beam penetrates the macromolecular

Received date: 2016-11-04 Accepted date: 2017-06-19

Biographies: Long Yuan, Master, research interests: application of terahertz technology, Email: longy@nercita.org.cn.

*Corresponding Author: Li Bin, $\mathrm{PhD}$, research interests: application of terahertz technology. Beijing Research Center for Information Technology in Agriculture, No.11, Shuguang Garden Road, Haidian District, Beijing 100097, China. Tel: +86-10-51503416, Fax: +86-10-51503750, Email: lib@ nercita.org.cn. substances, it will be attenuated due to the absorption of the substances and result in signal changes in the spectra, which makes it possible to do qualitative and quantitative analysis of the substances ${ }^{[2]}$. Moreover, the terahertz spectra can be used to detect the uncovering hidden macromolecular dynamics ${ }^{[3]}$. It had been applied into the detection of moisture in leaves ${ }^{[4]}$, identification of transgenic cottons ${ }^{[5]}$, detection and assessment of bacteria $^{[6]}$, determination of tetracycline's hydrochloride in infant formula milk powder ${ }^{[7]}$. Due to the influence of the intermolecular interactions, a terahertz $(\mathrm{THz})$ spectrum has different absorption peaks.

Norfloxacin could inhibit the replication of bacterial DNA and retard the growth of bacteria ${ }^{[8]}$. Norfloxacin-loaded collagen scaffolds can be used for 
skin reconstruction ${ }^{[9]}$. Norfloxacin can also be used for curing many diseases. For example, the rifampicin pharmacokinetics with norfloxacin on has positive effect on $\operatorname{man}^{[10]}$. The enzyme activities such as 7-ethoxycoumarin O-deethylase are inhibited with the increased norfloxacin concentrations ${ }^{[11]}$. As for the dendrimeric chelators with large molecular weights, their combination with norfloxacin may be applied to do treatments for the external infections ${ }^{[12]}$. With wide application of the norfloxacin into human, the detection of the norfloxacin is becoming more and more important. There are many ways to detect the norfloxacin such as flow injection analysis (FIA) differential spectrophotometer $^{[13]}$, ultraviolet spectrometry ${ }^{[14]}$ and fluorescence spectrophotometry ${ }^{[15]}$. However, these traditional detection methods are complex and their accuracies are limited ${ }^{[16-18]}$. Generally, these existing methods are mainly operated in the lab. Complex chemical analysis is needed and time-consuming (more than $12 \mathrm{~h}$ ). Also the instruments are very expensive and need special operation conditions. Accordingly, new rapid tools for qualitative and quantitative analysis of norfloxacin are in great need.

As norfloxacin is a macromolecular substance, it may have terahertz spectra fingerprint peaks in terahertz frequency domain. The aim of this study is to make an exploration for qualitative and quantitative analysis of norfloxacin based on terahertz spectroscopy. Norfloxacin samples making methods and experiments for acquiring $\mathrm{THz}$ spectra were designed respectively. Based on the collected data, mathematical models to describe the relationship between the terahertz spectra and norfloxacin content were established for the quantitative prediction.

\section{Materials and methods}

\subsection{Sample preparation}

The power of the instrument used in our experiment was limited and the terahertz spectrum was difficult to penetrate thicker samples. Therefore, the samples were made into tablets for further exploration. Polyethylene (PE) is one of the polymer plastics which are widely used in versatile applications, including $\mathrm{THz}$ technologies ${ }^{[19,20]}$. And it is largely transparent in the THz-region of the electromagnetic spectrum ${ }^{[21]}$. Polyethylene was used as a reference material in general, as it has no strong absorption features within the $\mathrm{THz}$ spectrum. As it is difficult to press the pure norfloxacin into tablets, the norfloxacin powder was mixed with polyethylene powder and then the mixture was put into the mould to make tablets with the tablet-making method. The particle size for norfloxacin and polyethylene pure solid powders (Sigma, USA) were ranged from $40 \mu \mathrm{m}$ to $48 \mu \mathrm{m}$. The proportions of norfloxacin in the mixture ranged from $10 \%$ to $90 \%$. Each sample was pressed with $8 \mathrm{MPa}$ for 3 min. Then the mixtures were pressed into round tablets with the diameter of $13 \mathrm{~mm}$. The thickness of the sample tablets ranged from $0.6 \mathrm{~mm}$ to $2 \mathrm{~mm}$. Forty-five samples in total were prepared. The detailed quality, thickness and proportion of the sample tablets in this study were shown in Table 1. Four of the tablets were shown in Figure 1.

\subsection{Terahertz spectroscopy instruments}

The terahertz spectroscopy instruments (TERA K15, Menlo Systems Company, Germany) were used in this study. The middle wavelength of the femtosecond laser is $1550 \mathrm{~nm}$. The repetition frequency is $100 \mathrm{MHz}$. The system used in the experiment is shown in Figure 2.

\subsection{Terahertz spectra data acquisition}

The experiment was conducted at room temperature. As for the strong absorption of moisture in terahertz wave, pure nitrogen gas was purged into the sealing cover consecutively to keep the humidity under $3 \%$ in order to reduce the moisture absorption in terahertz wave. The nitrogen reference spectra were collected prior to collecting the sample spectra to evaluate the system stability. After that, the terahertz spectroscopy instruments started to scan the sample; the $\mathrm{THz}$ spectra data were acquired. When performing the experiment, the terahertz spectra of three different points of each sample were collected. It took $5 \mathrm{~min}$ to scan a signal point on one tablet. Then the average spectra were calculated to represent the sample spectra in order to reduce the random error. 
Table 1 Sample tablets information

\begin{tabular}{|c|c|c|c|c|}
\hline Number & $P_{n} / \%$ & $M_{1} / \mathrm{mg}$ & $M_{0} / \mathrm{mg}$ & Thickness/mm \\
\hline 1 & 10 & \multirow{9}{*}{100} & 99.5 & 0.93 \\
\hline 2 & 20 & & 98.2 & 0.87 \\
\hline 3 & 30 & & 97.3 & 0.82 \\
\hline 4 & 40 & & 98.0 & 0.89 \\
\hline 5 & 50 & & 104.7 & 0.84 \\
\hline 6 & 60 & & 100.8 & 0.82 \\
\hline 7 & 70 & & 102.0 & 0.79 \\
\hline 8 & 80 & & 102.1 & 0.65 \\
\hline 9 & 90 & & 106.2 & 0.63 \\
\hline 10 & 10 & \multirow{9}{*}{150} & 147.4 & 1.29 \\
\hline 11 & 20 & & 149.6 & 1.30 \\
\hline 12 & 30 & & 154.8 & 1.23 \\
\hline 13 & 40 & & 153.0 & 1.23 \\
\hline 14 & 50 & & 142.6 & 1.05 \\
\hline 15 & 60 & & 149.8 & 1.04 \\
\hline 16 & 70 & & 151.9 & 1.03 \\
\hline 17 & 80 & & 154.1 & 0.95 \\
\hline 18 & 90 & & 152.0 & 0.89 \\
\hline 19 & 10 & \multirow{9}{*}{200} & 192.9 & 1.83 \\
\hline 20 & 20 & & 200.0 & 1.66 \\
\hline 21 & 30 & & 199.5 & 1.63 \\
\hline 22 & 40 & & 206.7 & 1.62 \\
\hline 23 & 50 & & 201.3 & 1.48 \\
\hline 24 & 60 & & 204.4 & 1.44 \\
\hline 25 & 70 & & 201.9 & 1.30 \\
\hline 26 & 80 & & 205.9 & 1.26 \\
\hline 27 & 90 & & 193.8 & 1.13 \\
\hline 28 & 10 & \multirow{9}{*}{125} & 117.2 & 1.10 \\
\hline 29 & 20 & & 124.5 & 1.08 \\
\hline 30 & 30 & & 123.4 & 0.98 \\
\hline 31 & 40 & & 123.7 & 1.04 \\
\hline 32 & 50 & & 124.4 & 0.98 \\
\hline 33 & 60 & & 123.8 & 0.89 \\
\hline 34 & 70 & & 125.1 & 0.92 \\
\hline 35 & 80 & & 124.4 & 0.81 \\
\hline 36 & 90 & & 129.1 & 0.81 \\
\hline 37 & 10 & \multirow{9}{*}{175} & 176.5 & 1.66 \\
\hline 38 & 20 & & 172.3 & 1.49 \\
\hline 39 & 30 & & 172.0 & 1.41 \\
\hline 40 & 40 & & 174.5 & 1.39 \\
\hline 41 & 50 & & 173.4 & 1.24 \\
\hline 42 & 60 & & 175.0 & 1.25 \\
\hline 43 & 70 & & 174.6 & 1.12 \\
\hline 44 & 80 & & 172.2 & 1.23 \\
\hline 45 & 90 & & 175.3 & 1.10 \\
\hline
\end{tabular}

Note: $P_{n}$ was the norfloxacin proportion in the mixture; $M_{1}$ is the mass of the mixture and $M_{0}$ is the mass of the tablets; Thickness refers to the tablets' thickness.

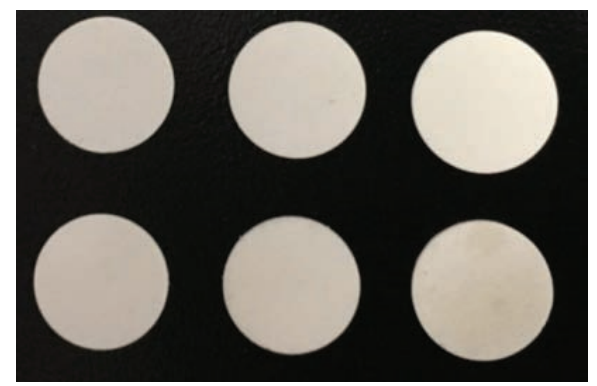

Figure 1 Norfloxacin tablets

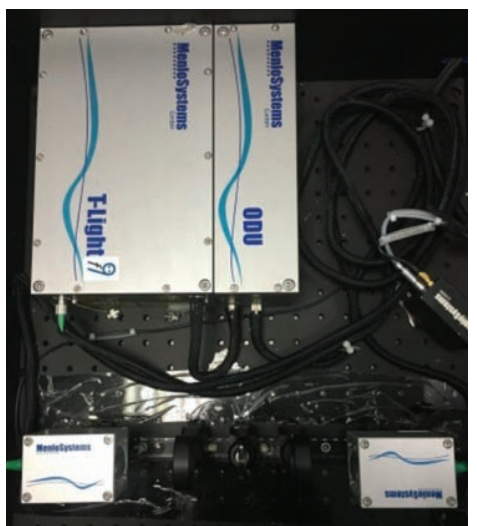

Figure 2 THz-TDS K15 system

\subsection{Statistics analysis}

The obtained raw spectra were time domain signal, which was ranged from 0-80 ps. Then corresponding frequency domain spectra were calculated by the fast Fourier transformation method from the time domain spectra. Then the absorption spectra $(a)$ and refractive spectra $(n)$ were calculated using standard algorithm ${ }^{[22-24]}$.

$$
\begin{gathered}
n=\frac{\left(\varphi_{S}(\omega)-\left(\varphi_{R}(\omega)\right) \times c\right.}{2 \pi f \times d} \\
a=\frac{1}{d} \times \ln \frac{A_{R}}{A_{S}}
\end{gathered}
$$

where, $\varphi_{S}(\omega)$ was the phase of the tablet spectral signal, and $\varphi_{R}(\omega)$ was the phase of the reference spectral signal. $c$ was the velocity of the light in a vacuum. And $f$ was the frequency of the tablet spectral signal, $d$ was the thickness of the tablet sample. $A_{S}$ and $A_{R}$ were the amplitude of the reference spectral signal and tablet sample spectral signal.

According to the fingerprint peaks, the multiple linear regressions ${ }^{[25]}$ and the partial least squares regression were used to build models to make a quantitative prediction about the norfloxacin respectively.

The correlation coefficient and the root mean square error of the calibration set and prediction set were calculated respectively to evaluate the accuracy of the models. The original spectra data were stored as TXT format and the data were processed with Matlab 2009 (MathWorks Inc, Natick, MA, USA).

\section{Results and discussion}

\subsection{Qualitative analysis of norfloxacin}

The time and frequency domain spectra of the norfloxacin tablets were shown in Figure 3. Compared 
with the reference spectra, the time and frequency domain spectra amplitude of the norfloxacin were attenuated as the result of the absorption of the norfloxacin to terahertz spectra. Meanwhile, the time domain spectra were delayed. As for the difference of the mixture proportion, the attenuation and the delay were different as the result of the different absorption. The THz-TDS system provides a time-domain signal that contains not only the initial pulse transmitted through the material but also several subsequent pulses, resulting from internal reflections, which arrive at delayed time ${ }^{[26]}$.

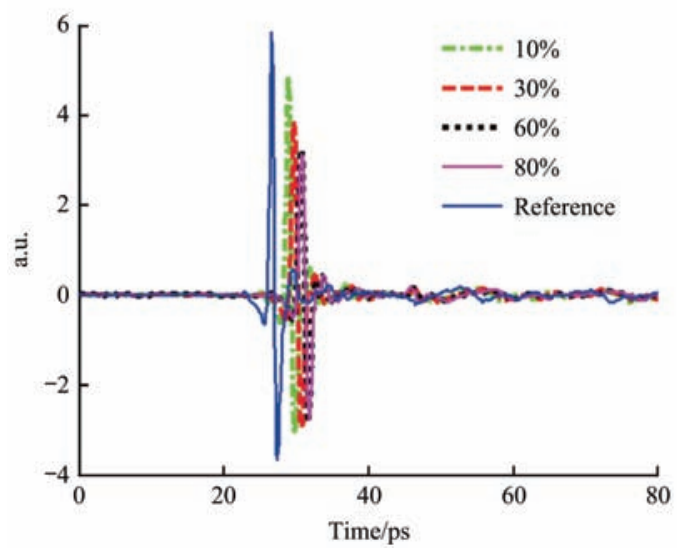

a. Time domain spectra

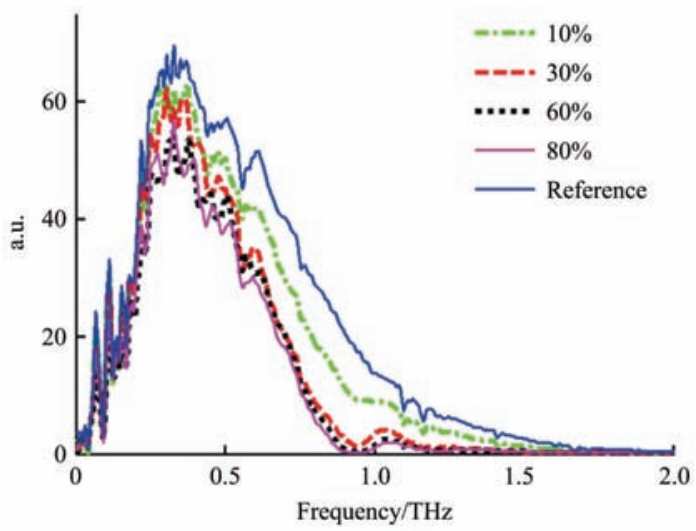

b. Frequency domain spectra

Note: Different line represents different concentration sample. The same below.

Figure 3 Terahertz time domain and frequency domain spectra

The absorption and refractive spectra were calculated respectively. Due to the large noise at both ends of the spectra, the signal-to-noise ratio (SNR) is low. Therefore, the spectra in the frequency from $0.2 \mathrm{THz}$ to 1.7 THz were selected for effective data. Two fingerprint peaks can be observed obviously. Terahertz spectra were absorbed strongly at $0.944 \mathrm{THz}$ and 1.206 $\mathrm{THz}$ (Figure 4). The two specific absorption peaks were the fingerprint peaks of norfloxacin in $\mathrm{THz}$ bands. The fingerprint peaks increased with the increase of the proportion of norfloxacin. The refractive index was used to describe the penetration characteristics of light in the medium. The refractive index of the norfloxacin tablets was ranged from 1.2 to 2.3 . The change of the refractive index was obvious in the frequency $0.944 \mathrm{THz}$ as the result of the terahertz absorption of the norfloxacin. From the above analysis, norfloxacin shows typical absorption peaks in $\mathrm{THz}$ band for qualitative analysis. Although the interaction is highly efficient, it should be noted that most of the generated $\mathrm{THz}$ waves are absorbed or totally reflected due to a large absorption coefficient, as well as a large refractive index in the THz range ${ }^{[27]}$.
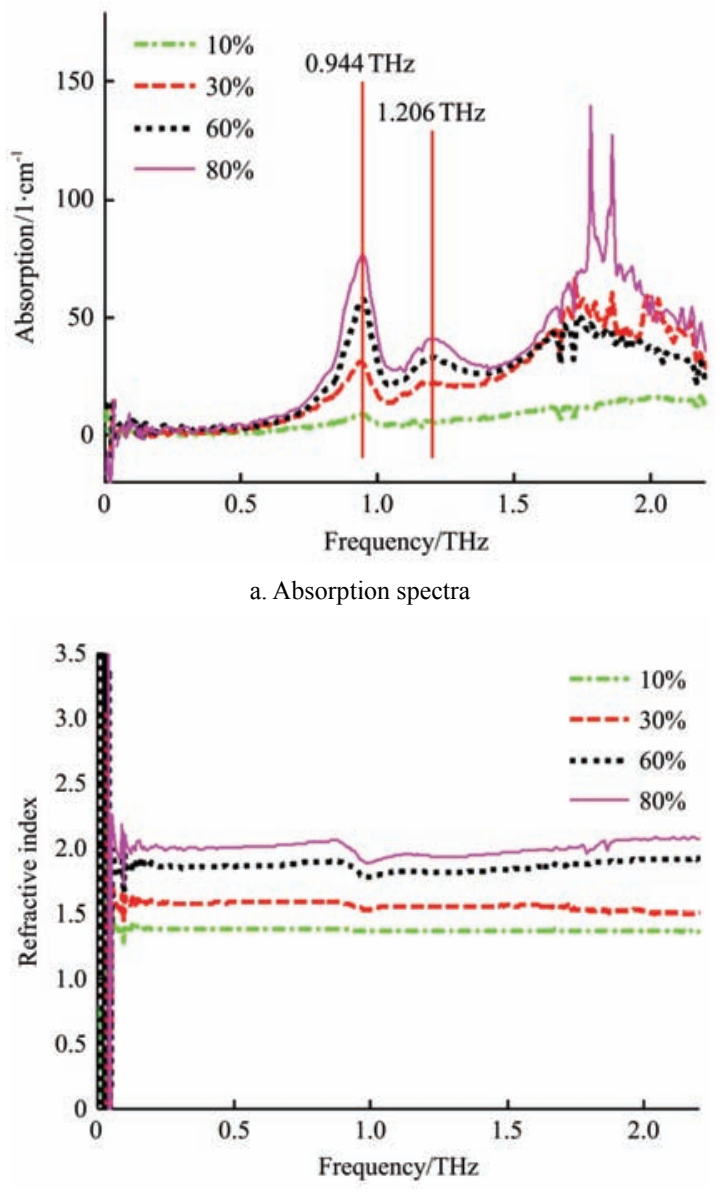

b. Refractive spectra

Figure 4 Terahertz absorption spectra and refractive spectra

\subsection{Quantitative analysis of norfloxacin}

In order to analyze the relationship between the terahertz spectra and norfloxacin content, the correlation coefficients between absorption spectra and norfloxacin concentration were calculated. As can be seen from Figure 5, the correlation coefficients at the low and large frequency were lower. At both ends, the fluctuations 
were larger as the result of the influence of the system noise. In the fingerprint peaks, the correlation coefficients had reached the peaks. It also can be concluded that the norfloxacin absorption peaks in the terahertz spectra were in the frequency of $0.944 \mathrm{THz}$ and 1.206 THz. The corresponding correlation coefficients were 0.9649 and 0.9028 , respectively. It should be noted that concentration of the norfloxacin caused the change of the peaks which will be effective for evaluating the norfloxacin quantitatively.

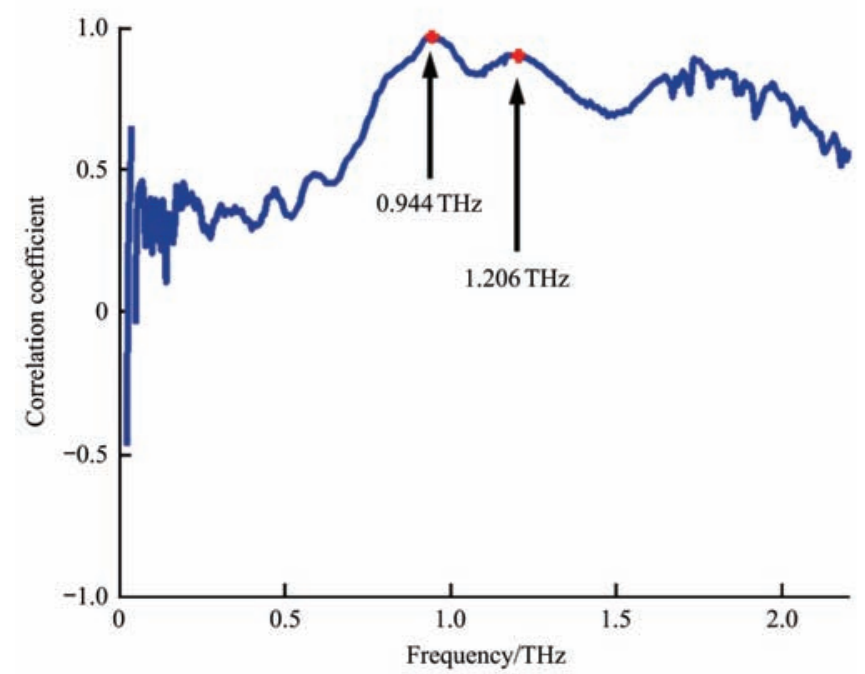

Figure 5 Correlation coefficient between absorption spectra and norfloxacin

\subsubsection{Multiple linear regressions}

Twenty-seven samples were classified into calibration set and the other 18 samples were selected as prediction set randomly. The models built with the multiple linear regressions were obtained. The two fingerprint peaks were used in the model as input because of the fingerprint peaks including most information about the $\mathrm{THz}$ spectra. The correlation coefficient and the root mean square error of the prediction set were 0.9651 and 0.0645 respectively. The results are shown in Table 2.

Table 2 Results of multiple linear regression

\begin{tabular}{ccccc}
\hline Wavelength & $R c$ & $R M S E C$ & $R p$ & $R M S E P$ \\
\hline $0.944 \mathrm{THz} \& 1.206 \mathrm{THz}$ & 0.9683 & 0.0684 & 0.9651 & 0.0645 \\
\hline
\end{tabular}

The multiple linear regression equation calculated was shown as follows:

$$
Y=-0.0241+0.0124 X_{0.944}-0.0038 X_{1.206}
$$

where, $Y$ was the concentration of the norfloxacin; $X_{0.944}$ and $X_{1.206}$ were the spectral signal at the frequency of $0.944 \mathrm{THz}$ and $1.206 \mathrm{THz}$ respectively.

\subsubsection{Partial least squares}

The multiple linear regressions were used with only information of the fingerprint peaks. Therefore, the model built with the multiple linear regressions may leave out some important information, indicating that more mathematical algorithms should be used to improve the prediction accuracy. The partial least squares regression models with different main factors were established with the whole wavelengths and it can reduce multiple-colinearity problem with the orthogonal transformation. The different models were built to get the optimal one. The whole terahertz bands were used to build the model with the partial least square. The first two main factors could represent the whole wavelength information. When the number of the main factors was more than two, the model was over fitted and the prediction accuracy decreased. The correlation coefficient and the root mean square error of the prediction set with two main factors were 0.9908 and 0.0481. The results of the partial least squares are shown in Table 3.

Table 3 Results of the partial least squares regression

\begin{tabular}{ccccc}
\hline Main factor & $R c$ & $R M S E C$ & $R p$ & $R M S E P$ \\
\hline 1 & 0.8901 & 0.1224 & 0.9061 & 0.1170 \\
2 & 0.9879 & 0.0427 & 0.9908 & 0.0481 \\
3 & 0.9895 & 0.0386 & 0.9852 & 0.0522 \\
4 & 0.9931 & 0.0318 & 0.9861 & 0.0485 \\
5 & 0.9941 & 0.0295 & 0.9785 & 0.0604 \\
6 & 0.9955 & 0.0258 & 0.9688 & 0.0604 \\
\hline
\end{tabular}

Comparing the results from multiple linear regressions and the partial least squares regression, the optimal model was the one that modeled by the partial least squares regression with two main factors (Figure 6). The partial least square regression model with the whole wavelengths include all the information in the $\mathrm{THz}$ spectra. In the meanwhile, the input spectral data were processed by orthogonal transformation in the partial least square regression and the prediction accuracy can be improved effectively. The accuracy of the model built with the partial least squares is higher than the model built with the multiple linear regressions. From what has been discussed above, it can be concluded that the norfloxacin content can be detected quantitatively in $\mathrm{THz}$ bands. 


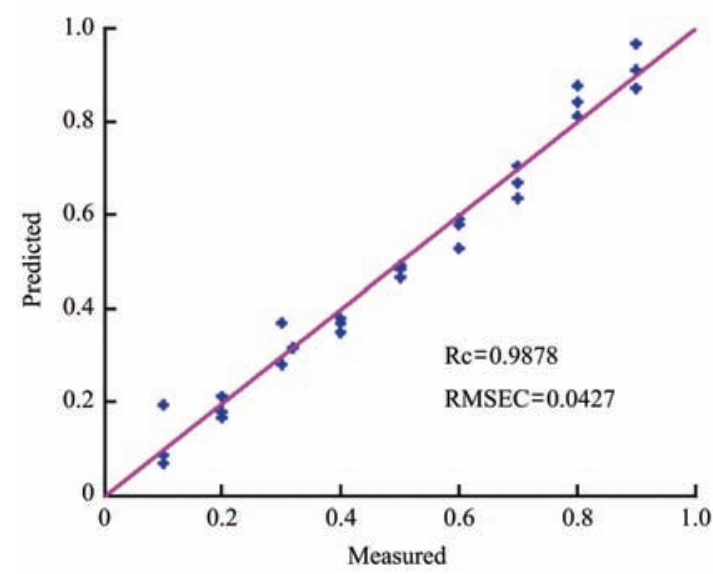

a. Calibration set

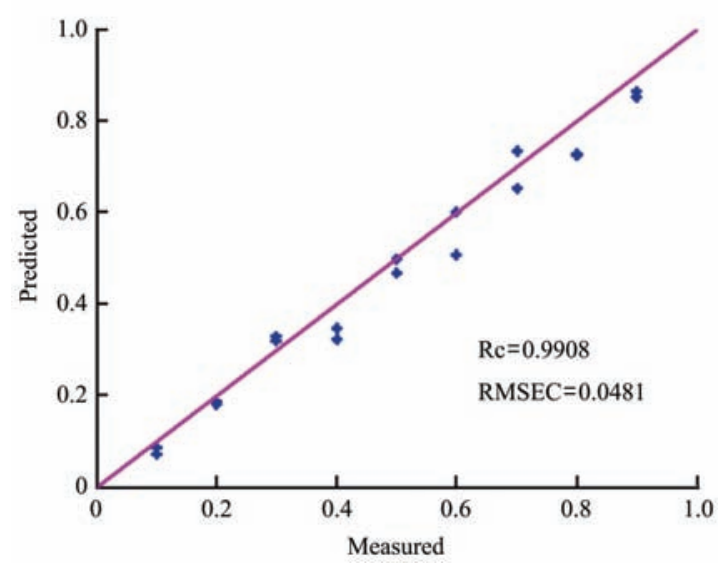

b. Prediction set

Figure 6 Scatter diagram of the optimal model

\section{Conclusions}

This preliminary study tried to make qualitative and quantitative analysis of norfloxacin by the terahertz spectroscopy. The norfloxacin terahertz spectra data were obtained by detecting the samples prepared by the tablet-making method. The fingerprint peaks were obtained at the frequency of $0.944 \mathrm{THz}$ and $1.206 \mathrm{THz}$ according to the absorption spectra. The multiple linear regressions and the partial least squares regressions were used to make a quantitative estimate for the norfloxacin. The correlation coefficient and the root mean square error of the best prediction set of the model that was established by the partial least squares regression method with two main factors were 0.9908 and 0.0481 , respectively. The model was relatively stable and its prediction accuracy was pretty high.

Compared with other methods, the $\mathrm{THz}$ detection method is very convenient and rapid. Terahertz spectroscopy is an emerging technology, which might be a potential way for analyzing norfloxacin qualitatively and quantitatively. Nevertheless, this study was preliminary and further study was needed to explore the maximum and minimum limit of the norfloxacin concentration. This preliminary study indicates that $\mathrm{THz}$ spectroscopy has great potential for future screening applications to detect the presence of norfloxacin residues in food, livestock feed and medicine industries.

\section{Acknowledgments}

This work was financially supported by the "Thirteenth Five-Year" National Key Research and Development Program of China (No. 2016YFD0702002) and the International Cooperation Fund of Beijing Academy of Agriculture and Forestry Sciences (GJHZ2017-7). Also we would like to thank THz Group for their support.

\section{[References]}

[1] John F F. Review of moisture and liquid detection and mapping using terahertz imaging. Journal of Infrared Millimeter and Terahertz Waves, 2012; 33(2): 97-126.

[2] Qin J Y, Ying Y B, Xie L J. The detection of agricultural products and food using terahertz spectroscopy: a review. Applied Spectroscopy Reviews, 2013; 48(6): 439-457.

[3] Rohit S, Deepu K G, Andrea M. Uncovering hidden macromolecular dynamics with modulated orientation sensitive terahertz spectroscopy. Quantitative Biology, 2012; 100(3): 225-228.

[4] Jördens C, Scheller M., Breitenstein B, Selmar D, Koch M. Evaluation of leaf water status by means of permittivity at terahertz frequencies. Journal of Biological Physics, 2009; 35(3): 255-264.

[5] Liu J J, Li Z, Hu F G, Chen T, Zhu A J, Du Y, et al. Method for identifying transgenic cottons based on terahertz spectra and WLDA. Optik, 2015; 126(19): 1872-1877.

[6] Yang X, Wei D S, Yan S H, Liu Y P, Shu Y, Zhang M K, et al. Rapid and label-free detection and assessment of bacteria by terahertz time-domain spectroscopy. Journal of Biophotonics, 2016; 9(10): 1050-1058.

[7] Qin J Y, Xie L J, Ying Y B. Feasibility of terahertz time-domain spectroscopy to detect tetracyclines hydrochloride in infant milk powder. Analytical Chemistry, 2014; 86(23): 11750-11757.

[8] Helmy S A. Simultaneous quantification of linezolid, tinidazole, norfloxacin, moxifloxacin, levofloxacin, and gatifloxacinin human plasma for therapeutic drug monitoring and pharmacokinetic studies in human volunteers. Therapeutic Drug Monitoring, 2013; 35(6): 770-777. 
[9] Azza A M, Alaa H S. Norfloxacin-loaded collagen/chitosan scaffolds for skin reconstruction: Preparation, evaluation and in-vivo wound healing assessment. European Journal of Pharmaceutical Sciences, 2016; 83: 155-165.

[10] Ndidi A E, Sinyeofori B, Ebenezer B, Orish E O. Effect of ofloxacin and norfloxacin on rifampicin pharmacokinetics in man. American Journal of Therapeutics, 2015; 22: 29-36.

[11] Li S W, Wang D, Liu H B, Lu T Y. Effects of norfloxacin on the drug metabolism enzymes of two sturgeon species. Journal of Applied Ichthyology, 2013; 29(6): 1204-1207.

[12] Zhou Y J, Zhang M X, Hider R C, Zhou T. In vitro antimicrobial activity of hydroxypyridinone hexadentate-based dendrimeric chelators alone and in combination with norfloxacin. FEMS Microbiology Letters, 2014; 355(2): 124-130.

[13] Han G X, Zhao Z Y, Yu H, Song X M. Determination of the content of norfloxacin capsule by FIA differential spectrophotometer. Chinese Journal of Pharmaceutical Analysis, 2008; 28(12): 2129-2131. (in Chinese)

[14] Tang Y H, Zhao Z X. Determination of norfloxacin capsule by ultraviolet spectrometry. Spectroscopy and Spectral Analysis, 1996; 16: 120-122.

[15] Cao F Q, Li D, Yan Z Y. Determination of norfloxacin by its enhancement effect on the fluorescence intensity of functionalized CdS nanoparticles. Spectroscopy and Spectral Analysis, 2009; 29(8): 2222-2226.

[16] Darwish I A, Blake D A. One-Step competitive immunoassay for cadmium ions: development and validation for environmental water samples. Analytical Chemistry, 2001; 73(8): 1889-1895.

[17] Beauchemin D. Inductively coupled plasma mass spectrometry. Journal of the American Society for Mass Spectrometry, 2007; 18(7): 1345.

[18] Kou S, Nam S W, Shumi W, Min H, Bae S W, Du J, et al.
Microfluidic detection of multiple heavy metal ions using fluorescent chemosensors. Bulletin of the Korean Chemical Society, 2009; 30(5): 1173.

[19] Wietzke S, Jansen C, Reuter M, Jung T, Hehl J, Kraft D, et al. Thermo morphological study of the terahertz lattice modes in polyvinylidene fluoride and high-density polyethylene. Applied Physics Letters, 2010; 97(2): 1-3.

[20] Yamamoto K, Yamagiichi M, Tani M, Hangyo M, Teramura $\mathrm{S}$, Isu $\mathrm{T}$, et al. Degradation diagnosis of ultra-highmolecular weight polyethylene (UHMWPE) by terahertz-time-domain spectroscopy. Applied Physics Letters, 2004; 85(22): 5194-5196.

[21] Palik E D. Handbook of Optical Constants Academic Press, 1985.

[22] Qin J Y, Xie L J, Ying Y B. Determination of tetracycline hydrochloride by terahertz spectroscopy with PLSR model. Food Chemistry, 2015; 170: 415-422.

[23] Timothy D D, Richard G B, Dnaiel M M. Material parameter estimation with terahertz time-domains spectroscopy. Journal of the Optical Society of America, 2001; 18(7): 1562-1571.

[24] Lionel D, Frederrie G, Jean L C. A reliable method for extraction of material parameters in terahertz time-domains spectroscopy. IEEE Journal of Selected Topic in Quantum Electronies, 2002; 2(3): 793-746.

[25] Altman N, Krzywinski M. Simple linear regression. Nature Methods, 2015; 12(11): 999-1000.

[26] Exter M V, Fattinger C, Grischkowsky D. Terahertz time-domain spectroscopy of water vapor. Optics Letters, 1989; 14(20): 1128-1130.

[27] Nazarov M, Shkurinov A, Tuchin V, Zhang X. Terahertz Tissue Spectroscopy and Imaging. Handbook of Photonics for Biomedical Science, 2010; 5: 519-617. 